\title{
Evolution of Intracavitary Brachytherapy in the Treatment of Cervical Cancer
}

\section{Chrishanthi Rajasooriyar ${ }^{1,2 *}$ and Peter Hoskin ${ }^{3,4}$}

${ }^{1}$ Teaching Hospital, Jaffna, Sri Lanka

${ }^{2}$ Tellipalai Trail Cancer Hospital, Jaffna, Sri Lanka

${ }^{3}$ Mount Vernon Cancer Centre, Northwood, United Kingdom

${ }^{4}$ University of Manchester, Manchester, United Kingdom

*Corresponding Author: Chrishanthi Rajasooriyar, Teaching Hospital, Jaffna, Sri

Lanka.
Received: April 05, 2021

Published: June 30, 2021

(C) All rights are reserved by Chrishanthi

Rajasooriyar and Peter Hoskin.

\section{Abstract}

Purpose: The evolution of intracavitary brachytherapy in the treatment of cervical cancer spans over 120 years. The purpose of this review is to understand how the discovery of radium paved the way to the current standard of care; the image-guided adaptive brachytherapy.

Materials and Methods: A literature search was done to identify the important milestones in the evolution. The terminologies used for the search were "history", "brachytherapy", "radium", "dosimetric systems" and "image-guided adaptive brachytherapy" (IGABT). The information gathered was organized in chronological order and the key milestones were identified.

Results: The origin of brachytherapy can be tracked down to the discovery of radioactivity by Sir Henry Becquerel, followed by the invention of radium by the Curies' in the late $19^{\text {th }}$ century. In the early $20^{\text {th }}$ century, various dosimetric systems evolved. Among these systems, the Manchester system prescribing to point A became popular and was widely in practice for many decades. The deficiencies of the point A prescription model were brought to light with the advances in soft tissue imaging in the late $20^{\text {th }}$ century. Imaging helped in prescribing to the actual tumor volume and to generate dose-volume histograms to achieve better target coverage while reducing the dose to the organs at risk. The IGABT started with computed tomography and was further enhanced by magnetic resonance imaging (MRI) due to better anatomical delineation of the pelvis anatomy. Hence MRI based adaptive brachytherapy became the gold standard and has been proven to be safe and effective while significantly reduced toxicity. As cervix cancer is most prevalent in low resource regions, ultrasound scanning has been identified as a reasonable substitute for MRI.

Conclusion: Intracavitary brachytherapy in the treatment of cervical cancer has developed over the last 120 years and has made tremendous advances in the last 20 years.

Keywords: Cervix Cancer; Intracavitary Brachytherapy; Image-guided Adaptive Brachytherapy

\section{Introduction}

Brachytherapy in combination with external beam radiotherapy (EBRT) comprises the definitive treatment of locally advanced cervix cancer.
The tumoricidal dose of radiation needed to achieve good local control of cervix cancer is estimated between 80 - 90Gy [1]. A typical course of external beam irradiation will deliver 45-50Gy beyond which normal tissue tolerances may be exceeded resulting 
in significant toxicity. This is overcome by using brachytherapy to deliver radiation directly into the tumour bearing region. Intracavitary brachytherapy has been identified to be the most important treatment factor predictive of survival and pelvic control in the curative setting [2]. A survival benefit has also been confirmed in a population-based study [3] and, the use of brachytherapy has been recommended in all cervix cancer patients treated with definitive radiotherapy with or without concurrent chemotherapy [3].

Recent declines in the use of brachytherapy have resulted in reduced overall survival and local control ${ }^{4}$ and attempts to replace brachytherapy with a Stereotactic Ablative Body Radiation Therapy (SABR) boost have demonstrated increased higher-grade toxicity and worse local control and survival [5].

The history and evolution of gynecological brachytherapy span over a century. The clinical use of brachytherapy using radium began at the beginning of the $20^{\text {th }}$ century prior to which surgery was the solely available treatment for cervix cancer. The first use of intracavitary radium in the treatment of cervix cancer was reported by Margaret Cleaves in 1903 [6]. The honour of curing the first patient with cervix cancer using radium brachytherapy goes to Sir Robert Abbe [7]; this milestone being achieved in 1905.

Brachytherapy use was temporarily abandoned from 1910 to 1920 due to high cost, World War I, resistance from the surgeons, radiation toxicity, and above all the perception of a temporary nature of cure based on the low success rates [7].

\section{The birth of dosimetric systems}

Dosimetry in brachytherapy follows the inverse square law accounting for dose decay away from the source. A number of different point based dosimetric systems were developed with their own specific design of applicators. These included the Stockholm, Paris, Manchester and Munich systems [8] in Europe and the Fletcher system in the United States of America [7].

The Manchester system used three different uterine sources that best matched the length of the uterus and three different sizes of ovoids that best fitted the vagina. The source strength of the uterine tubes was 20,25 , and $35 \mathrm{mg}$ of radium for uterine applicators measuring 2,4 and $6 \mathrm{~cm}$ respectively. The source activities of the ovoids were $17.5,20$, and $22.5 \mathrm{mg}$ of radium for small, medium, and large ovoids respectively [9]. This system recommended two fractions of brachytherapy with each lasting for 72 hours at 10 days gap. The patients' anatomy determined the selection of the size of the uterine tube and the vaginal ovoids needed for the application. This system became very popular and was commonly practiced around the globe for more than seven decades with minimum modifications.

With access to routine radiology, it became clear when comparing the actual doses at points $\mathrm{A}$ and $\mathrm{B}$ based on pelvic $\mathrm{X}$-rays with the precalculated doses at point $A$ and $B$, there was a significant difference in the doses at actual vs pre-calculated points $A$ and $B$. The need for orthogonal x-rays following the insertion of brachytherapy applicators to understand the actual doses received by the patient [10] was clear. This led to the development of $\mathrm{x}$-ray based dosimetry.

The Fletcher system evolved as a modification of the Paris and the Manchester systems [11]. This system used three intrauterine sources of $10 \mathrm{mg}, 10 \mathrm{mg}, 15 \mathrm{mg}$ of radium, and two vaginal colpostats containing $20 \mathrm{mg}$ each [8]. Fletcher had a broader understanding of the deficiencies of the brachytherapy system that existed and highlighted the problems associated with 'points-based prescription' when volumes of tissue being subjected to treatment. He also considered the importance of dose to OAR and stressed the need for dose measurement in the bladder and rectum. He further emphasized the importance of dose measurements in the para-cervical triangle and the regional nodes to compensate for underdosing with EBRT [12].

\section{The transition from LDR to HDR brachytherapy}

With the move from manual insertions to remote after loading there was also an increase in dose rate, initially using caesium which approximately doubled the dose rate from radium, 0.4Gy per hour to around 1 Gy per hour, still in the range of low-medium dose rate but requiring a dose reduction. The major change however was the move to remote after loading with caesium and subsequently high dose rate sources such as cobalt and iridium. The added advantage of iridium was the high specific activity which meant that sources were smaller than before, typically around $2 \mathrm{~mm}$ diameter. Such small sources significantly reduced treatment time which minimized the risk of displacement of the applicator during treatment and made day care procedures possible [13].

Multiple comparisons between HDR and LDR have been published, including meta-analysis which confirmed similar outcomes 
[14] provided appropriate adjustments to total dose and fractionation were employed.

A need for guidelines recommending a uniform approach for prescription and reporting was recognized and the International Commission on Radiation Units and Measurements (ICRU) published recommendations in 1985 (ICRU 38) [15]. This defined the prescription point at Point A, $2 \mathrm{~cm}$ lateral to the midline and above the superior surface of the vaginal source. Point $B$ was defined 3 $\mathrm{cm}$ lateral to Point A and specific dosimetry points for bladder and rectal reporting were defined as shown in figure 1.

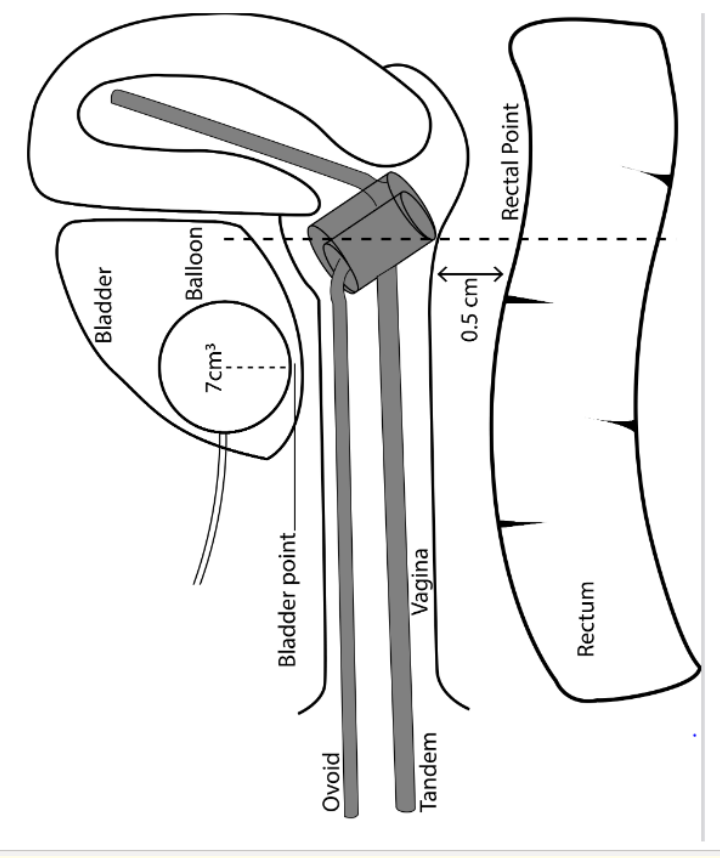

Figure 1

The introduction of soft tissue imaging highlighted the deficiencies of Point A based dosimetry, particularly when defined using a plain x-ray. Using CT and later MRI, it was possible to identify tumor bulk and shape and normal tissues. It became clear that point A can be lying either inside or outside the target volume leading to overdosing or underdosing of the target volume [16]. Similarly it became clear that the ICRU bladder and rectal points were inconsistent and did not represent any specific dosimetric measure such as maximum or mean dose and that there was considerable individual variation.

Figure 2 shows an implant that is ideal on orthogonal X-ray with ideal Tandem length and tandem in mid-position between sacrum and pubis, bisecting the ovoids. However, the MRI of the same patient reveals that the tandem is not in the ideal position and there is underdosing of the target volume. Figure 3 shows an implant that is anatomically sound but not radiologically sound as seen in the orthogonal film. Figure 4 shows three orientations of a plan normalized to Point A. The axial view highlights the amount of sigmoid in the high dose zone. The coronal image highlights Point A in normal tissue on the right side of the uterus and Point $A$ in sigmoid on the left side of the uterus. Sagittal image shows encroachment of $100 \%$ isodose into the bladder. Hence, it was obvious that the 'pear' needed sculpting.

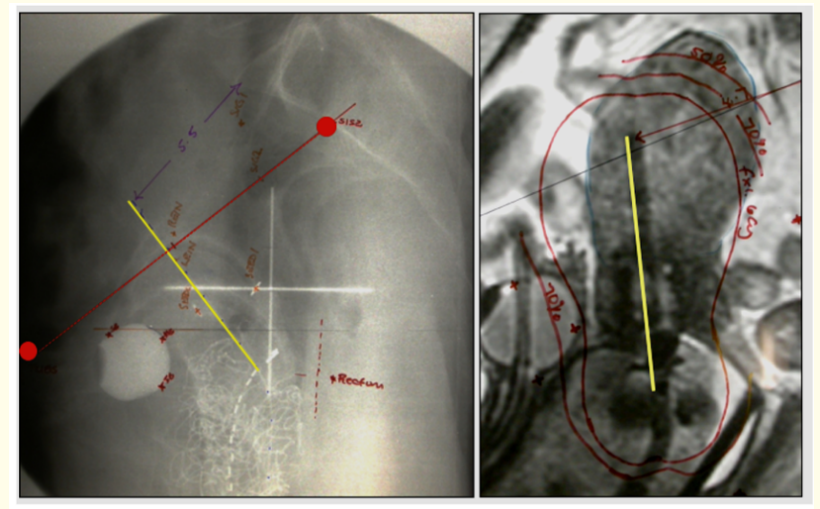

Figure 2

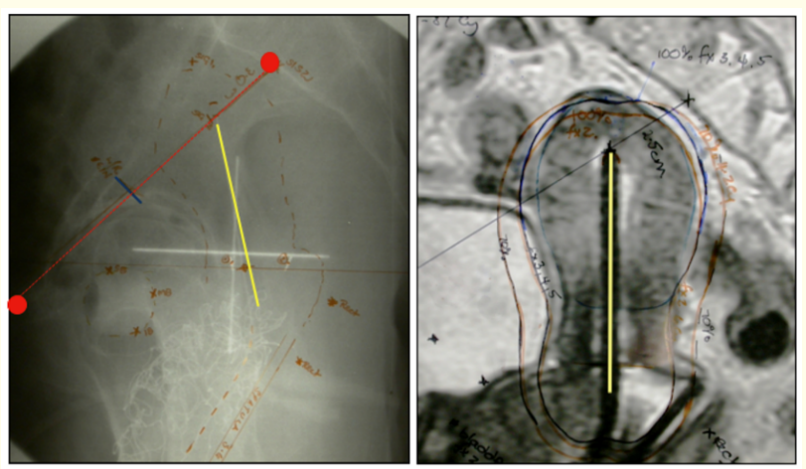

Figure 3 


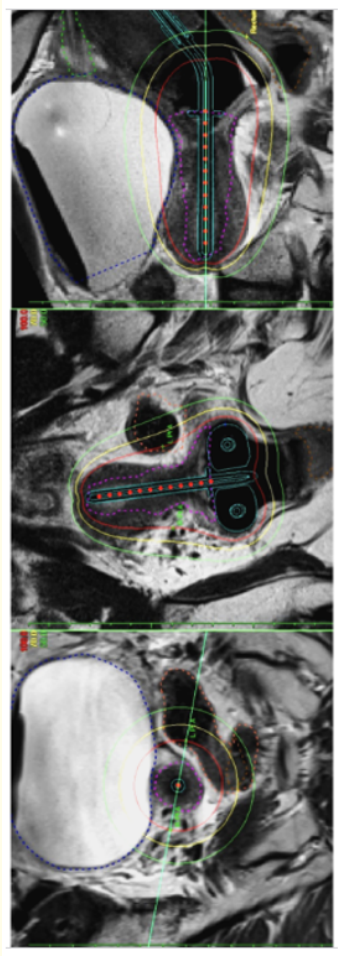

Figure 4

Importance of technical quality of the implant

The technical quality of brachytherapy in patients with stages I -III cervix cancer treated with curative intent radiotherapy is important. A statistically significant difference in 5-year local control has been shown when good quality implants (68\%) were compared to poor quality implants (34\%) [17]. Technical adequacy was defined by lack of symmetry and displacement of the colpostats. The colpostats were expected to be placed at equal distance from the tandem and the most inferior tandem source and the colpostat sources were expected to be in the same plane. Multivariate analysis identified the technical competence of the brachytherapy application to be the single most important prognostic factor leading to better local control [17]. The American Brachytherapy Society consensus guidelines also emphasize the importance of precise applicator placement to optimise therapeutic index [18].

Advanced soft tissue imaging revolutionizing brachytherapy planning

CT based brachytherapy planning was introduced in the early nineties with the invention of artifact-free applicators. Many stud- ies have compared the target coverage and dose to OAR in both conventional point A based brachytherapy with CT based 3 dimensional planning and confirmed better dosimetry in terms of target coverage and OAR doses with CT based planning [19-25].

The soft tissue delineation of pelvic anatomy is superior with MRI compared to CT scans [26]. Hence, MRI based adaptive brachytherapy gained momentum with the development of MRI compatible applicators. The superiority of MRI over CT in brachytherapy planning was confirmed in published literature [27-29].

A number of planning studies soon demonstrated the advantage of 3D planning over point based planning both in terms of target coverage and organ at risk doses [20,25].

The expanding use of image-guided brachytherapy in cervix cancer necessitated the development of recommendations for clinical application and terminology for reporting. Independent but complimentary guidelines from the Groupe Européen de $\mathrm{Cu}$ riethérapie and the European Society for Radiotherapy and Oncology (GEC-ESTRO) working group and the American brachytherapy Society (ABS IGBWG) were published. There were some differences in detail for example outlining of the rectum as a single cylinder (GEC ESTRO) or a doughnut (ABS) but overall there was general agreement and these guidelines have now been incorporated into ICRU Report 89 [30]. The guidelines provide clear recommendations for delineation of targets such as "gross target volume (GTV), high-risk clinical target volume (HR-CTV), intermediate-risk clinical target volume (IR-CTV), and OARs (rectum, bladder, sigmoid colon, and any adjacent bowel loops)". The GTV, HR-CTV, and IR-CTV denote a regressing density of malignant cells needing declining doses of radiation [30] as shown in figure 5.

\section{The success story of MRI guided adaptive brachytherapy}

Excellent clinical outcomes with concurrent chemoradiotherapy and MRI guided adaptive brachytherapy have been reported in a multi-institutional cohort (RetroEMBRACE study) with a 5 year local control, pelvic control, cause-specific survival and overall survival of $89 \%, 84 \%, 65 \%$ and $73 \%$ respectively with limited severe morbidity [31].

The results of the RetroEMBRACE (Retro European study of MR based imaging in locally advanced cervical cancer) study was compared with a similar subgroup of patients of the STIC trial which 


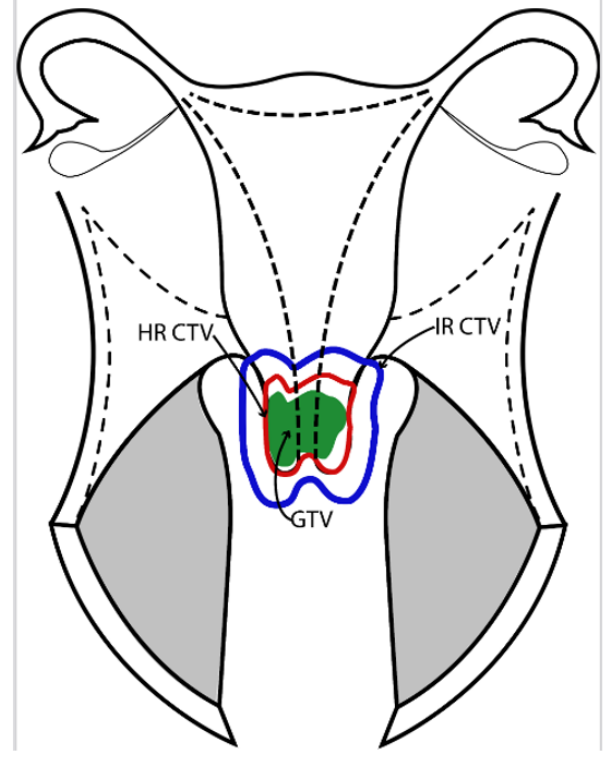

Figure 5 compared the impact of 2D vs 3D brachytherapy [23]. The comparison showed better loco-regional control with a $20 \%$ reduction in grade 3-4 toxicity favouring the 3D technique [25,31,32].

The GEC-ESTRO recommendations for MRI based brachytherapy planning have been widely adopted and the benefits have been well proven [33].

Limited resource setting alternative for MRI guided adaptive brachytherapy

Whilst MR based IGBT with daily imaging before each fraction of HDR delivery is the gold standard in practice this is not feasible even in many developed countries. It has been shown that CT can replace MR for second and subsequent fractions [34] and ultrasound has great potential providing real time imaging within the brachytherapy environment. Cervix cancer is one of the most common cancers in the developing world and practicing MRI based brachytherapy is challenging due to high patient burden and limitations in the availability of advanced imaging options and insufficient human resources. An alternative method of image-guided brachytherapy uses ultrasound imaging. Comparison of both MRI and ultrasound (US) imaging in 192 patients resuting in 1668 measurements of the cervix and uterus showed that the variation in the measurements of the cervix and uterus on MRI and US were within clinically acceptable limits [35]. This study recommended the use of trans-abdominal US as a substitute for MRI in conformal brachytherapy treatment planning. Further, the same group from Melbourne published their clinical outcomes with single MRI and the serial US for planning and target verification and reported outcomes equivalent to MRI/CT based planning [36]. Similar correlation between trans rectal ultrasound and MRI in assessing the target has been reported [37]. Hence, there is good evidence that US could replace MRI in resource-limited settings to still achieve better outcomes.

\section{Conclusion}

Intracavitary brachytherapy in the treatment of cervical cancer has evolved over the last 120 years and made tremendous advances in the last 20 years. MRI based adaptive brachytherapy is the current standard of care. Alternative options are available for resource-limited settings.

\section{Conflict of Interest}

The author has none to declare.

\section{Bibliography}

1. Nag S., et al. "The American Brachytherapy Society recommendations for high-dose-rate brachytherapy for carcinoma of the cervix". International Journal of Radiation Oncology • Biology • Physics 48.1 (2000): 201-211.

2. Lanciano RM., et al. "Pretreatment and treatment factors associated with improved outcome in squamous cell carcinoma of the uterine cervix: A final report of the 1973 and 1978 patterns of care studies". International Journal of Radiation Oncology • Biology • Physics 20.4 (1991): 667-676.

3. Han K., et al. "Trends in the utilization of brachytherapy in cervical cancer in the United States". International Journal of Radiation Oncology • Biology • Physics 87.1 (2013): 111-119.

4. Schad MD., et al. "Declining brachytherapy utilization for cervical cancer patients - Have we reversed the trend?" Gynecologic Oncology 156.3 (2020): 583-590. 
5. Albuquerque K., et al. "A Phase II Trial of Stereotactic Ablative Radiation Therapy as a Boost for Locally Advanced Cervical Cancer". International Journal of Radiation Oncology • Biology • Physics 106.3 (2020): 464-471.

6. Cleaves MA. "Radium: With a preliminary note on radium rays in the treatment of cancer". Medication Reconciliation 64 (1903): 601-606.

7. Mould RF. "The Historical Roots of Modern Brachytherapy for Cervical and Endometrial Cancer". Radiation Oncology of Gynecological Cancers (1997): 1-9.

8. Evolution T. "3. Brachytherapy Techniques and Systems". Journal of the International Commission on Radiation Units 13 (2013): 21-35.

9. TOD M and MEREDITH WJ. "Treatment of cancer of the cervix uteri, a revised Manchester method". British Journal of Radiology 26.305 (1953): 252-257.

10. G C Lewis Jr and A Raventos JH. "Space Dose Relationships for Points A and B in the Radium Therapy of Cancer of the Uterine Cervix". American Journal of Roentgenology Radium Therapy and Nuclear Medicine 83 (1960): 432-446.

11. Erickson BA. "The sculpted pear: An unfinished brachytherapy tale". In: Brachytherapy. (2003).

12. FLETCHER., et al. "Direct measurements and isodose calculations in radium therapy of carcinoma of the cervix". Radiology 61.6 (1953): 885-902.

13. Romano KD., et al. "Transition from LDR to HDR brachytherapy for cervical cancer: Evaluation of tumor control, survival, and toxicity". Brachytherapy 16.2 (2017): 378-386.

14. Gaur R., et al. "Brachytherapy in Carcinoma Cervix: Results From a Randomized Study". 23.4 (2012).

15. ICRU Report 38. "Dose and volume specification for reporting intracavitary therapy in gynecology". Journal of the International Commission on Radiation Units (1985).

16. Pötter R., et al. "Present status and future of high-precision image guided adaptive brachytherapy for cervix carcinoma”. Acta Oncologica (Madr). 47.7 (2008): 1325-1336.
17. Corn BW., et al. "Technically accurate intracavitary insertions improve pelvic control and survival among patients with locally advanced carcinoma of the uterine cervix". Gynecologic Oncology 53.3 (1994): 281-384.

18. Viswanathan AN and Thomadsen B. "American Brachytherapy Society consensus guidelines for locally advanced carcinoma of the cervix. Part I: General principles". Brachytherapy (2012).

19. Fellner C., et al. "Comparison of radiography- and computed tomography-based treatment planning in cervix cancer in brachytherapy with specific attention to some quality assurance aspects". Radiotherapy and Oncology 58.1 (2001): 53-62.

20. Shin KH., et al. "CT-guided intracavitary radiotherapy for cervical cancer: Comparison of conventional point a plan with clinical target volume-based three-dimensional plan using dose-volume parameters". International Journal of Radiation Oncology • Biology • Physics 64.1 (2006): 197-204.

21. Simpson DR., et al. "Clinical outcomes of computed tomography-based volumetric brachytherapy planning for cervical cancer". International Journal of Radiation Oncology • Biology • Physics 93.1 (2015): 150-157.

22. Thomas KM., et al. "Reduced toxicity with equivalent outcomes using three-dimensional volumetric (3DV) image-based versus nonvolumetric point-based (NV) brachytherapy in a cervical cancer population". Brachytherapy 16.5 (2017): 943-948.

23. Charra-Brunaud C., et al. "Impact of 3D image-based PDR brachytherapy on outcome of patients treated for cervix carcinoma in France: Results of the French STIC prospective study". Radiotherapy and Oncology 103.3 (2012): 305-313.

24. Derks K., et al. "Impact of brachytherapy technique (2D versus 3D) on outcome following radiotherapy of cervical cancer". Journal of Contemporary Brachytherapy 10.1 (2018): 17-25.

25. Madan R., et al. "Comparative evaluation of two-dimensional radiography and three dimensional computed tomography based dose-volume parameters for high-dose-rate intracavitary brachytherapy of cervical cancer: A prospective study". Asian Pacific Journal of Cancer Prevention 15.11 (2014): 47174721. 
26. Hriack H., et al. "Early Invasive Cervical Cancer: CT and MR Imaging in Preoperative Evaluation". Radiology 245.2 (2007): 491-498.

27. Viswanathan AN., et al. "Computed Tomography Versus Magnetic Resonance Imaging-Based Contouring in Cervical Cancer Brachytherapy: Results of a Prospective Trial and Preliminary Guidelines for Standardized Contours". International Journal of Radiation Oncology • Biology • Physics 68.2 (2007): 491-498.

28. Eskander RN., et al. "Comparison of computed tomography and magnetic resonance imaging in cervical cancer brachytherapy target and normal tissue contouring". International Journal of Gynecological Cancer 20.1 (2010): 47-53.

29. Krishnatry R., et al. "CT or MRI for image-based brachytherapy in cervical cancer". Japanese Journal of Clinical Oncology 42.4 (2012): 309-313.

30. Europe G. "Prescribing, Recording, and Reporting Brachytherapy for Cancer of the Cervix". Journal of the International Commission on Radiation Units 13 (2013): 1-10.

31. Sturdza A., et al. "Image guided brachytherapy in locally advanced cervical cancer: Improved pelvic control and survival in RetroEMBRACE, a multicenter cohort study". Radiotherapy and Oncology 120.3 (2016): 428-433.

32. Venkat PS., et al. "Outcome Assessment of Dose Adaptation of MRI-Guided Cervical Cancer Brachytherapy". International Journal of Radiation Oncology (2014).

33. Haie-Meder C., et al. "Recommendations from Gynaecological (GYN) GEC-ESTRO Working Group (I): Concepts and terms in 3D image based 3D treatment planning in cervix cancer brachytherapy with emphasis on MRI assessment of GTV and CTV". Radiotherapy Oncology 74.3 (2005): 235-245.

34. Nesvacil N., et al. "Adaptive image guided brachytherapy for cervical cancer: A combined MRI-/CT-planning technique with MRI only at first fraction". Radiotherapy Oncology 107.1 (2013): 75-81.

35. Van Dyk S., et al. "Comparison of measurements of the uterus and cervix obtained by magnetic resonance and transabdominal ultrasound imaging to identify the brachytherapy target in patients with cervix cancer". International Journal of Radiation Oncology • Biology • Physics 88.4 (2014): 860-865.
36. van Dyk S., et al. "Clinical outcomes from an innovative protocol using serial ultrasound imaging and a single MR image to guide brachytherapy for locally advanced cervix cancer". Brachytherapy 15.6 (2016): 817-824.

37. Schmid MP., et al. "Feasibility of transrectal ultrasonography for assessment of cervical cancer". Strahlentherapie und Onkologie 189.2 (2013): 123-128.

\section{Volume 3 Issue 7 July 2021 \\ (C) All rights are reserved by Chrishanthi Rajasooriyar and Peter Hoskin.}

